\title{
Assessment of Right Liver Lobe Size / Serum Albumin Ratio as a New Non-Invasive Predictor for the Presence of Oesophageal Varices in Egyptian Patients with HCV Related Liver Cirrhosis
}

\author{
Emad Ahmad Awad, Wael Ahmed Yousry, Hasan Mokhtar Hasan, Ahmed Mohamed EIGhandour* \\ Department of Internal Medicine, Gastroenterology and Hepatology, Faculty of Medicine, \\ Ain Shams University, Cairo, Egypt \\ *Correspondence to Dr. Ahmed Mohamed ElGhandour, E-mail: ghandour80@ hotmail.com, \\ Telephone number: 00201001973493
}

\begin{abstract}
Background: Oesophageal varices are the most critical porto-systemic shunts that develop secondary to portal hypertension, which is considered the main complication of liver cirrhosis. Many studies recommend the screening of all cirrhotic patients by endoscopy, but repeated endoscopic examinations are unpleasant for patients and have a highcost impact and burden on endoscopic units. Objective: This study aimed to evaluate the accuracy of using the right liver lobe size/serum albumin ratio as a non-invasive predictor of esophageal varices in patients with HCV-related liver cirrhosis. Patients Methods: This prospective study included 30 patients with liver cirrhosis and 30 patients who underwent upper gastrointestinal endoscopy for any causes other than liver cirrhosis. All studied subjects underwent a detailed history and clinical examination, biochemical workup, upper gastrointestinal endoscopy, and abdominal ultrasound. The right liver lobe/serum albumin ratio was calculated for all patients.

Results: There was a statistically significant difference between the control and the study subgroups as regards the Right lobe of the Liver/Albumin ratio (p-value 0.007). The diagnostic accuracy of the Right lobe of the Liver/Albumin ratio was assessed using the ROC curve which revealed a sensitivity of $86.67 \%$ and specificity of $73.33 \%$ at cut-off value $>3.88$, with an acceptable discriminative accuracy of $79.9 \%$.

Conclusion: The use of Right liver lobe/serum albumin ratio can help physicians by restricting the use of endoscopic screening only to patients presenting a high probability of esophageal varices. This is especially useful in clinical settings where resources are limited, and endoscopic facilities are not present in all areas.
\end{abstract}

Keywords: Non-invasive diagnosis of oesophageal varices, Right liver lobe size/serum albumin ratio, Oesophageal varices, $\mathrm{HCV}$.

\section{INTRODUCTION}

Esophageal varices are submucosal, expanding esophageal veins connecting the portal and systemic circuits ${ }^{(\mathbf{1})}$. This is caused by portal hypertension most commonly due to cirrhosis, resistance to portal blood flow, and increased inward portal venous blood flow (2). The most common fatal complication of cirrhosis is the rupture of varicose veins. The severity of the liver disease is associated with the presence of varicose veins and the risk of bleeding ${ }^{(3,4)}$. Variceal bleeding occurs in $20-40 \%$ of cirrhotic patients with esophageal varices and is associated with high morbidity and mortality ${ }^{(5)}$. Mortality associated with each episode of varicose veins ranges from $17 \%$ to $57 \%{ }^{(6)}$.

Gastroesophageal endoscopy (EGD) is the gold standard procedure used in the diagnosis of esophageal varices (GOVs). Based on the endoscopic evaluation, GOVs are classified into small $(<5 \mathrm{~mm})$ and large $(>5 \mathrm{~mm})$ varicose veins for clinical management. The disadvantages of endoscopy include the risks of anesthesia, high cost, bleeding, and risk of aspiration (7). Endoscopic ultrasound (EUS) has also been evaluated as a diagnostic tool in the evaluation of GOVs. Endoscopic ultrasound imaging is better than EGD at detecting gastric varices $(\mathrm{GVs})$, and its ability to evaluate the anatomy of the lateral and perforate veins makes it an excellent choice in monitoring treatment response to endoscopic varicose ligation (EVL) and predicting their recurrence ${ }^{(\mathbf{8}, \mathbf{9})}$. Currently, EUS is not considered a primary diagnostic method due to the Limited availability of local expertise. A recent meta-analysis reviewed the use of capsule endoscopy for the diagnosis and classification of esophageal varices and noted a diagnostic accuracy of $90 \%$ with a combined sensitivity and specificity of $83 \%$ and $85 \%$, respectively ${ }^{(\mathbf{1 0})}$.

The inability of capsule endoscopy to detect GVs is a major drawback. Although capsule endoscopy is relatively less invasive and does not require anesthesia, diagnostic sensitivity is not enough to call for index monitoring. There may be a consideration for a select subset of high-risk patients who do not wish to undergo further conventional endoscopic evaluation (11, 12). For these reasons, several studies have examined how to identify patients with varicose veins using non-invasive or minimally invasive methods to avoid endoscopy in patients with low risk of varicose veins. However, it is not accurate enough to diagnose or exclude clinically significant portal hypertension (CSPH) (HVPG> $12 \mathrm{~mm} \mathrm{Hg}$ ). Specifically, transient 
elastography, platelet count, spleen volume, magnetic resonance imaging, and splenosclerosis are among the most frequently used predictors of CSPH and varicose veins in patients with cirrhosis. The presence of jihadist portal collaterals on ultrasound, computed tomography, or magnetic resonance imaging is indicative of $\mathrm{CSPH}$ and requires an endoscopic examination ${ }^{(7)}$.

Measurement of liver stiffness using transient elastography combined with a platelet count can rule out the presence of high-risk varicose veins ${ }^{(13)}$. Hepatic stiffness $<20 \mathrm{kPa}$ and platelet count $>150,000$ $/ \mu \mathrm{l}$ indicates a less than $5 \%$ chance of developing high-risk varicose veins, and endoscopic examination can be safely postponed if continuous clinical monitoring can be assured ${ }^{(\mathbf{1 4})}$. The use of endoscopic prophylactic tape ligation and non-selective betablockers can reduce the risk of esophageal bleeding by $50 \%{ }^{(15)}$. Therefore, the guidelines recommend that all patients with cirrhosis be screened by endoscopy to identify those at risk of bleeding so that they can be administered prophylactic (14). Practice guidelines issued by the American College of Gastroenterology (ACG) and the American Association for the Study of Liver Diseases (AASLD) for esophageal varices indicate that all cirrhotic patients should be screened for varicose veins at least every two years ${ }^{(16)}$.

In this study, we aim to investigate noninvasive predictors of esophageal varices in patients with liver cirrhosis using the right liver lobe/serum albumin ratio as a non-invasive predictor of esophageal varices in cirrhotic patients, for restricting the performance of screening endoscopy.

\section{PATIENTS AND METHODS}

This cross-sectional study was conducted on sixty patients who were selected from Internal Medicine and Hepatology outpatient clinics and inpatient wards at Ain shams University Hospitals. After eligibility was confirmed, written informed consent was obtained from all patients participating in the study. Patients were divided into 2 groups:

Group 1 (cases): which included 30 age and sexmatched cirrhotic patients due to $\mathrm{HCV}$ infection with or without liver cell failure. The diagnosis was based on physical findings, laboratory investigations, and ultrasonographic findings.

Group 2 (control): This included 30 subjects attending the endoscopy unit for conditions other than cirrhosis.

Patients with previous variceal bleeding, previous endoscopic sclerotherapy or band ligation of oesophageal varices, previous surgery for portal hypertension or trans jugular, intrahepatic, portosystemic shunt (TIPS), patients those taking Beta-blocker (e.g, propanolol or carvedilol) medications, patients with other causes of liver cirrhosis, patients with portal vein thrombosis or
HCC, other conditions that may affect serum albumin level, serum platelet, liver or spleen size (e.g. diabetes mellitus, renal disease, cancer, malnutrition, malabsorption syndrome, infections, patients on regular albumin therapy and patients with hematological diseases), also other causes of hepatomegaly as fatty liver, congested liver or infiltration of the liver (by e.g, lymphoma or sarcoidosis) were excluded from the study.

\section{All participants were subjected to:}

\section{- Full history taking and clinical examination.}

\section{- Laboratory investigations included:}

- Complete Blood Count (CBC).

- Liver function tests: Alanine Transaminase (ALT), Aspartate Transaminase (AST), Serum Bilirubin (total, direct \& indirect), Serum Albumin, and Prothrombin Time (PT) and activity, alfa fetoprotein.

- Hepatitis viral markers e.g. Hepatitis B surface Antigen (HBsAg), Hepatitis C Virus antibody ( $\mathrm{HCV} \mathrm{Ab).}$

- Renal function tests including serum creatinine, urea, and urine analysis.

- Random blood sugar.

- Abdominal Ultrasound: patients were prepared for abdominal ultrasound by Hitachi, EUB-5500 after fasting for eight hours. One investigator performed all measurements to reduce the interobserver errors in assessing the diameters. Abdominal ultrasound included:

(A) Diagnosis of liver cirrhosis or normal liver.

(B) Excluding other diseases of the liver rather than cirrhosis and any disease of other organs in the abdomen.

(C) Ultrasound measurement of the right liver lobe diameter $(\mathrm{cm})$ :

While the patient is in the supine position a curvilinear probe of a high-resolution real-time scanner was placed sub-costally in the right midclavicular line in a sagittal plane showing a good liver window. The craniocaudal right lobe diameter at the mid-clavicular line was measured from the diaphragm to the liver edge. Measurements were taken during deep inspiration to reduce masking by the lung, with the right hand under the head to raise the lower costal margin.

(D) Ultrasound measurement of the spleen diameter (cm):

While the patient is in the right lateral decubitus position with the left arm raised away from the abdomen, the transducer was placed between the ribs at the level of the ninth intercostal space, and then the patient was asked to take a deep breath and hold it. The transducer was manipulated in the coronal plane or the coronal oblique plane until a suitable longitudinal view of the spleen is obtained, then the length of the spleen was measured between the 
superior and the inferior borders of the spleen. Gel was applied to the upper abdomen before scanning for better resolution.

- Right liver lobe size /serum Albumin ratio was calculated.

- Platelet count/spleen diameter ratio was calculated.

- Upper GI endoscopy to assess the presence of esophageal varices.

\section{Statistical analysis}

Data were collected, coded, translated to English to facilitate data manipulation and double entered Microsoft Access, and data analysis was performed using SPSS software version 18 under windows 10.

\section{RESULTS}

This cross-sectional study involved 60 patients made of two groups, HCV cirrhotic patients (study group) and non-cirrhotic patients who came for endoscopy for any other causes than liver cirrhosis (control group) each comprising 30 patients.

As regards the epidemiological features of our patients their ages were ranged as follows: Control group ranged from 33-69 years with a mean of $48 \pm$ 10.51 (Males represent $50 \%$ and females represent $50 \%$ also), while the Study group ranged from $45-61$ years with a mean of $53 \pm 4.60$ (Males represents $46.67 \%$ and females represent $53.33 \%$ ).

Table (1): Comparison between control \& study groups as regard to laboratory investigations.

\begin{tabular}{|c|c|c|c|c|c|c|c|c|}
\hline \multirow[b]{3}{*}{ AST (U/L) } & \multicolumn{6}{|c|}{ Groups } & \multicolumn{2}{|c|}{ T-Test } \\
\hline & \multicolumn{3}{|c|}{$\begin{array}{c}\text { Control } \\
\mathbf{N}=30\end{array}$} & \multicolumn{3}{|c|}{$\begin{array}{l}\text { Study } \\
\text { N=30 }\end{array}$} & \multirow{2}{*}{$\begin{array}{c}\mathbf{T} \\
-2.183\end{array}$} & \multirow{2}{*}{$\begin{array}{l}\text { P-value } \\
<0.001^{*}\end{array}$} \\
\hline & 26 & \pm & 8.54 & 58.5 & \pm & 10.16 & & \\
\hline ALT (U/I) & 25.5 & \pm & 8.76 & 56 & \pm & 9.12 & -2.211 & $<0.001 *$ \\
\hline Total Bilirubin (mg/dl) & 0.9 & \pm & 0.20 & 3.1 & \pm & 0.56 & -3.468 & $<0.001 *$ \\
\hline Direct Bilirubin (mg/dl) & 0.2 & \pm & 0.01 & 1.4 & \pm & 0.24 & -4.217 & $<0.001 *$ \\
\hline Indirect Bilirubin (mg/dl) & 0.75 & \pm & 0.24 & 1.7 & \pm & 0.04 & -3.125 & $<0.001 *$ \\
\hline Serum Albumin (g/dl) & 3.53 & \pm & 0.42 & 2.66 & \pm & 0.07 & 5.326 & $<0.001 *$ \\
\hline Prothrombin time "PT" (sec) & 12.17 & \pm & 1.54 & 15.91 & \pm & 3.00 & -4.775 & $<0.001 *$ \\
\hline INR & 1.01 & \pm & 0.02 & 1.5 & \pm & 0.08 & -6.661 & $<0.001 *$ \\
\hline Urea (mg/dl) & 13.5 & \pm & 2.89 & 15 & \pm & 2.30 & -3.505 & $<0.005$ \\
\hline Creatinine(mg/dl) & 0.8 & \pm & 0.14 & 1 & \pm & 0.14 & -0.470 & 0.640 \\
\hline White blood cells "WBCs" $\left(\mathrm{x}^{3} \mathbf{3}^{3} / \mathrm{ml}^{3}\right)$ & 6.95 & \pm & 1.78 & 6.22 & \pm & 1.04 & -3.574 & 0.624 \\
\hline Red blood cells "RBCs" $\left(\mathrm{x10}^{3} / \mathrm{ml}^{3}\right)$ & 4.11 & \pm & 0.87 & 3.85 & \pm & 0.50 & 3.250 & 0.051 \\
\hline Haemoglobin "Hb" (g/dl) & 10.8 & \pm & 1.94 & 10.25 & \pm & 0.58 & 1.985 & 0.052 \\
\hline Alpha fetoprotein (ng/mL) & 5.5 & \pm & 2.51 & 75.23 & \pm & 17.54 & -21.778 & $<0.001 *$ \\
\hline Platelet $\left(\mathrm{x10}^{3} / \mathrm{ml}^{3}\right)$ & 238.10 & \pm & 8.16 & 109.47 & \pm & 19.82 & 7.882 & $<0.001 *$ \\
\hline Right liver lobe (cm) & 13.240 & \pm & 1.257 & 15.357 & \pm & 0.837 & -4.050 & $<0.005$ \\
\hline Spleen diameter $(\mathrm{cm})$ & 12.910 & \pm & 1.744 & 16.543 & \pm & 1.793 & -7.955 & $<0.001 *$ \\
\hline Platelet count /spleen diameter ratio & 18.44 & \pm & 7.50 & 6.62 & \pm & 1.04 & 8.570 & $<0.001 *$ \\
\hline Right liver lobe size / Serum Albumin ratio & 3.750 & \pm & 0.295 & 5.773 & \pm & 0.809 & -3.728 & $<0.005$ \\
\hline
\end{tabular}

This table showed that there were highly statistically significant difference between the cirrhotic liver group (study group) and the non-cirrhotic group (control group) as regard AST, ALT, Total bilirubin, direct bilirubin, indirect bilirubin, serum albumin, PT, INR, Alpha-fetoprotein (p-value0.001), also there were highly statistically significant difference between the two groups as regard spleen diameter, platelet count ( $\mathrm{p}$-value0.001). But there were highly statistically significant differences between both groups as regard Right liver lobe size/Serum Albumin ratio and Right Liver Lobe (p-value <0.005).

Table (2): Grades of OV in the study group

\begin{tabular}{|l|c|c|}
\hline \multicolumn{1}{|c|}{ Study groups } & Number & \% \\
\hline Grade I varices (Group I) & 6 & 10.00 \\
\hline Grade II varices (Group II) & 8 & 13.33 \\
\hline Grade III varices (Group III) & 9 & 15.55 \\
\hline Grade IV varices (Group IV) & 7 & 11.67 \\
\hline Total & 30 & 100.00 \\
\hline
\end{tabular}

Table 2 showed the grading of oesophageal varices in the study group. 
Table (3): Comparison of right liver lobe size between the control group and subgroups of the study group

\begin{tabular}{|c|c|c|c|c|c|}
\hline \multirow{2}{*}{ Groups } & \multicolumn{3}{|c|}{ Right lobe of Liver } & \multicolumn{2}{|c|}{ ANOVA } \\
\hline & Mean & \pm & SD & $\mathbf{F}$ & P-value \\
\hline $\begin{array}{l}\text { Control } \\
\mathrm{N}=\mathbf{3 0}\end{array}$ & 13.240 & \pm & 1.257 & \multirow{5}{*}{3.971} & \multirow{5}{*}{$0.007 *$} \\
\hline $\begin{array}{l}\text { Group I } \\
\mathrm{N}=6\end{array}$ & 14.870 & \pm & 0.925 & & \\
\hline $\begin{array}{l}\text { Group II } \\
\mathrm{N}=8\end{array}$ & 14.938 & \pm & 0.780 & & \\
\hline $\begin{array}{l}\text { Group III } \\
\mathbf{N}=9\end{array}$ & 14.922 & \pm & 0.743 & & \\
\hline $\begin{array}{l}\text { Group IV } \\
\mathrm{N}=7\end{array}$ & 14.886 & \pm & 1.085 & & \\
\hline \multicolumn{6}{|c|}{ TUKEY'S Test } \\
\hline & \multicolumn{2}{|c|}{ Control } & Group I & Group II & Group III \\
\hline Group I & \multicolumn{2}{|c|}{0.089} & & & \\
\hline Group II & \multicolumn{2}{|c|}{0.163} & 0.992 & & \\
\hline Group III & \multicolumn{2}{|c|}{0.047} & 1.000 & 0.997 & \\
\hline Group IV & \multicolumn{2}{|c|}{0.168} & 0.997 & 1.000 & 0.999 \\
\hline
\end{tabular}

There was a statistically significant difference between subgroups of the study group as regard Rt liver lobe and the grade of O.V.

Table (4): Comparison between the control group and study subgroups as regard serum albumin

\begin{tabular}{|c|c|c|c|c|c|c|}
\hline \multirow{2}{*}{ Groups } & \multicolumn{3}{|c|}{ Serum Albumin } & \multicolumn{3}{|c|}{ ANOVA } \\
\hline & Mean & \pm & SD & $\mathbf{F}$ & \multicolumn{2}{|c|}{ P-value } \\
\hline $\begin{array}{l}\text { Control } \\
\mathbf{N}=\mathbf{3 0}\end{array}$ & 3.440 & \pm & 0.561 & \multirow{5}{*}{5.985} & \multirow{5}{*}{\multicolumn{2}{|c|}{$<0.001 *$}} \\
\hline $\begin{array}{l}\text { Group I } \\
\mathrm{N}=6\end{array}$ & 3.150 & \pm & 0.339 & & & \\
\hline $\begin{array}{l}\text { Group II } \\
\mathrm{N}=8\end{array}$ & 2.913 & \pm & 0.264 & & & \\
\hline $\begin{array}{l}\text { Group III } \\
\mathrm{N}=9\end{array}$ & 2.789 & \pm & 0.298 & & & \\
\hline $\begin{array}{l}\text { Group IV } \\
\mathrm{N}=7\end{array}$ & 2.714 & \pm & 0.530 & & & \\
\hline \multicolumn{7}{|c|}{$\begin{array}{l}\text { TUKEY'S Test } \\
\end{array}$} \\
\hline & \multicolumn{2}{|r|}{ Control } & \multicolumn{2}{|r|}{ Group I } & Group II & Group III \\
\hline $\begin{array}{l}\text { Group I } \\
\mathrm{N}=6\end{array}$ & \multicolumn{3}{|c|}{0.658} & & & \\
\hline $\begin{array}{l}\text { Group II } \\
\mathrm{N}=8\end{array}$ & \multicolumn{3}{|c|}{0.056} & 0.888 & & \\
\hline $\begin{array}{l}\text { Group III } \\
\mathrm{N}=9\end{array}$ & \multicolumn{3}{|c|}{0.006} & 0.609 & 0.984 & \\
\hline $\begin{array}{l}\text { Group IV } \\
\mathrm{N}=7\end{array}$ & \multicolumn{2}{|r|}{0.006} & & 0.480 & 0.929 & 0.998 \\
\hline
\end{tabular}

This table showed a highly statistically significant difference between the control group and the study subgroups according to $\mathrm{O} . \mathrm{V}$ grades as regard serum albumin level (p-value 0.001 ) 
Table (5): Comparison between the control group and study subgroups as regard platelet count

\begin{tabular}{|c|c|c|c|c|c|}
\hline \multirow{2}{*}{ Groups } & \multicolumn{3}{|c|}{ Platelet count } & \multicolumn{2}{|c|}{ ANOVA } \\
\hline & Mean & \pm & SD & $\mathbf{F}$ & P-value \\
\hline $\begin{array}{l}\text { Control } \\
\mathrm{N}=30\end{array}$ & 238.100 & \pm & 8.162 & \multirow{5}{*}{15.717} & \multirow{5}{*}{$<0.001 *$} \\
\hline $\begin{array}{l}\text { Group I } \\
\mathrm{N}=6\end{array}$ & 135.667 & \pm & 19.086 & & \\
\hline $\begin{array}{l}\text { Group II } \\
\mathrm{N}=8 \\
\end{array}$ & 113.125 & \pm & 7.376 & & \\
\hline $\begin{array}{l}\text { Group III } \\
\mathrm{N}=9\end{array}$ & 106.000 & \pm & 12.062 & & \\
\hline $\begin{array}{l}\text { Group IV } \\
\mathrm{N}=7\end{array}$ & 87.286 & \pm & 6.473 & & \\
\hline \multicolumn{6}{|c|}{ TUKEY'S Test } \\
\hline & Control & & Group I & Group II & Group III \\
\hline $\begin{array}{l}\text { Group I } \\
\mathrm{N}=6\end{array}$ & $0.006^{*}$ & & & & \\
\hline $\begin{array}{l}\text { Group II } \\
\mathrm{N}=8\end{array}$ & $<0.001^{*}$ & & 0.965 & & \\
\hline $\begin{array}{l}\text { Group III } \\
\mathrm{N}=9\end{array}$ & $<0.001 *$ & & 0.902 & 0.999 & \\
\hline $\begin{array}{l}\text { Group IV } \\
\mathrm{N}=7\end{array}$ & $<0.001 *$ & & 0.654 & 0.935 & 0.977 \\
\hline
\end{tabular}

This table showed that there was a highly statistically significant difference between the control group and study subgroups as regard platelet count ( $\mathrm{p}$-value $<0.001$ ).

Table (6): Comparison between the control group and study subgroups as regard spleen diameter

\begin{tabular}{|c|c|c|c|c|c|}
\hline \multirow{2}{*}{ Groups } & \multicolumn{3}{|c|}{ Spleen diameter } & \multicolumn{2}{|c|}{ ANOVA } \\
\hline & Mean & \pm & SD & $\mathbf{F}$ & P-value \\
\hline $\begin{array}{l}\text { Control } \\
\mathbf{N}=\mathbf{3 0} \\
\end{array}$ & 12.910 & \pm & 1.744 & \multirow{5}{*}{36.722} & \multirow{5}{*}{$<0.001 *$} \\
\hline $\begin{array}{l}\text { Group I } \\
\mathrm{N}=6\end{array}$ & 14.433 & \pm & 1.250 & & \\
\hline $\begin{array}{l}\text { Group II } \\
\mathrm{N}=8\end{array}$ & 15.788 & \pm & 0.530 & & \\
\hline $\begin{array}{l}\text { Group III } \\
\mathrm{N}=9\end{array}$ & 16.611 & \pm & 0.426 & & \\
\hline $\begin{array}{l}\text { Group IV } \\
N=7\end{array}$ & 19.129 & \pm & 0.808 & & \\
\hline \multicolumn{6}{|c|}{ TUKEY'S Test } \\
\hline & \multicolumn{2}{|c|}{ Control } & Group I & Group II & Group III \\
\hline $\begin{array}{l}\text { Group I } \\
\mathrm{N}=6\end{array}$ & \multicolumn{2}{|c|}{0.109} & & & \\
\hline $\begin{array}{l}\text { Group II } \\
\mathrm{N}=8\end{array}$ & \multicolumn{2}{|c|}{$<0.001 *$} & 0.368 & & \\
\hline $\begin{array}{l}\text { Group III } \\
\mathbf{N = 9}\end{array}$ & \multicolumn{2}{|c|}{$<0.001 *$} & $0.031^{*}$ & 0.730 & \\
\hline $\begin{array}{l}\text { Group IV } \\
N=7\end{array}$ & \multicolumn{2}{|c|}{$<0.001 *$} & $<0.001 *$ & $<0.001 *$ & $0.005^{*}$ \\
\hline
\end{tabular}

There was a highly statistically significant difference between the control group and study subgroups as regard the spleen diameter ( $\mathrm{p}$-value $<0.001$ ) 
Table (7): Comparison between the control group and study subgroups as regard Right lobe of Liver/Albumin ratio

\begin{tabular}{|c|c|c|c|c|c|}
\hline \multirow{2}{*}{ Groups } & \multicolumn{3}{|c|}{ Right lobe of Liver/Albumin ratio } & \multicolumn{2}{|c|}{ ANOVA } \\
\hline & Mean & \pm & SD & $\mathbf{F}$ & P-value \\
\hline $\begin{array}{l}\text { Control } \\
\mathbf{N}=30\end{array}$ & 4.048 & \pm & 1.295 & \multirow{5}{*}{3.927} & \multirow{5}{*}{$0.007 *$} \\
\hline $\begin{array}{l}\text { Group I } \\
\mathrm{N}=6\end{array}$ & 4.657 & \pm & 0.637 & & \\
\hline $\begin{array}{l}\text { Group II } \\
\mathrm{N}=8 \\
\end{array}$ & 4.930 & \pm & 0.600 & & \\
\hline $\begin{array}{l}\text { Group III } \\
\mathrm{N}=9\end{array}$ & 5.230 & \pm & 0.664 & & \\
\hline $\begin{array}{l}\text { Group IV } \\
\mathrm{N}=7\end{array}$ & 5.451 & \pm & 1.189 & & \\
\hline \multicolumn{6}{|c|}{ TUKEY'S Test } \\
\hline & Control & & Group I & Group II & Group III \\
\hline $\begin{array}{l}\text { Group I } \\
\mathrm{N}=6\end{array}$ & \multicolumn{2}{|l|}{0.722} & & & \\
\hline $\begin{array}{l}\text { Group II } \\
\mathrm{N}=8\end{array}$ & \multicolumn{2}{|l|}{0.263} & 0.990 & & \\
\hline $\begin{array}{l}\text { Group III } \\
\mathrm{N}=9\end{array}$ & \multicolumn{2}{|l|}{0.046} & 0.855 & 0.979 & \\
\hline $\begin{array}{l}\text { Group IV } \\
\mathrm{N}=7\end{array}$ & \multicolumn{2}{|l|}{0.026} & 0.685 & 0.886 & 0.994 \\
\hline
\end{tabular}

Table 7 showed that there was a statistically significant difference between the control and the study subgroups as regards the Right lobe of Liver/Albumin ratio (p-value 0.007).

Table (8): Comparison between the control group and study subgroups as regard Platelet count/Spleen diameter ratio

\begin{tabular}{|c|c|c|c|c|c|}
\hline \multirow{2}{*}{ Groups } & \multicolumn{3}{|c|}{ Platelet count/Spleen diameter ratio } & \multicolumn{2}{|c|}{ ANOVA } \\
\hline & Mean & \pm & SD & $\mathbf{F}$ & P-value \\
\hline $\begin{array}{l}\text { Control } \\
\mathrm{N}=30\end{array}$ & 18.981 & \pm & 7.505 & \multirow{5}{*}{18.975} & \multirow{5}{*}{$<0.001 *$} \\
\hline $\begin{array}{l}\text { Group I } \\
\mathrm{N}=6\end{array}$ & 9.575 & \pm & 2.447 & & \\
\hline $\begin{array}{l}\text { Group II } \\
\mathrm{N}=8\end{array}$ & 7.180 & \pm & 0.612 & & \\
\hline $\begin{array}{l}\text { Group III } \\
\mathrm{N}=9\end{array}$ & 6.387 & \pm & 0.734 & & \\
\hline $\begin{array}{l}\text { Group IV } \\
\mathrm{N}=7\end{array}$ & 4.563 & \pm & 0.295 & & \\
\hline \multicolumn{6}{|c|}{ TUKEY'S Test } \\
\hline & & Control & Group I & Group II & Group III \\
\hline $\begin{array}{l}\text { Group I } \\
\mathrm{N}=6\end{array}$ & \multicolumn{2}{|r|}{$0.003^{*}$} & & & \\
\hline $\begin{array}{l}\text { Group II } \\
\mathrm{N}=8\end{array}$ & \multicolumn{2}{|r|}{$<0.001 *$} & 0.928 & & \\
\hline $\begin{array}{l}\text { Group III } \\
\mathrm{N}=9\end{array}$ & \multicolumn{2}{|r|}{$<0.001^{*}$} & 0.807 & 0.998 & \\
\hline $\begin{array}{l}\text { Group IV } \\
\mathrm{N}=7\end{array}$ & \multicolumn{2}{|r|}{$<0.001 *$} & 0.482 & 0.889 & 0.965 \\
\hline
\end{tabular}

There was a highly statistically significant difference between the control group and the study subgroups as regard Platelet count/Spleen diameter ratio ( $\mathrm{p}$-value <0.001) 
Table (9): Statistical analysis of Right lobe of Liver/Albumin ratio and Platelet count/Spleen diameter ratio between Study and Control groups for ROC curve

\begin{tabular}{|l|c|c|c|c|c|c|}
\hline \multicolumn{7}{|c|}{ Statistical Indices } \\
\hline & Cutoff & Sens. & Spec. & PPV & NPV & Accuracy \\
\hline Right lobe of Liver/Albumin ratio & $>3.88$ & $\mathbf{8 6 . 6 7}$ & $\mathbf{7 3 . 3 3}$ & $\mathbf{7 6 . 5}$ & $\mathbf{8 4 . 6}$ & $\mathbf{7 9 . 9 \%}$ \\
\hline Platelet count/Spleen diameter ratio & $\leq 1415$ & $\mathbf{9 6 . 6 7}$ & $\mathbf{8 3 . 3 3}$ & $\mathbf{8 5 . 3}$ & $\mathbf{9 6 . 2}$ & $\mathbf{9 3 . 4 \%}$ \\
\hline
\end{tabular}

The diagnostic accuracy of the Right lobe of Liver/Albumin ratio was assessed using the ROC curve which revealed a sensitivity of $86.67 \%$ and specificity of $73.33 \%$ at cut-off value $>3.88$, with an acceptable discriminative accuracy of 79.9\%, also the diagnostic accuracy of Platelet count/Spleen diameter ratio was assessed using ROC curve which revealed a sensitivity $96.67 \%$ and specificity of $83.33 \%$ at cut-off value $\leq 1415$, with an acceptable discriminative accuracy of $93.4 \%$.

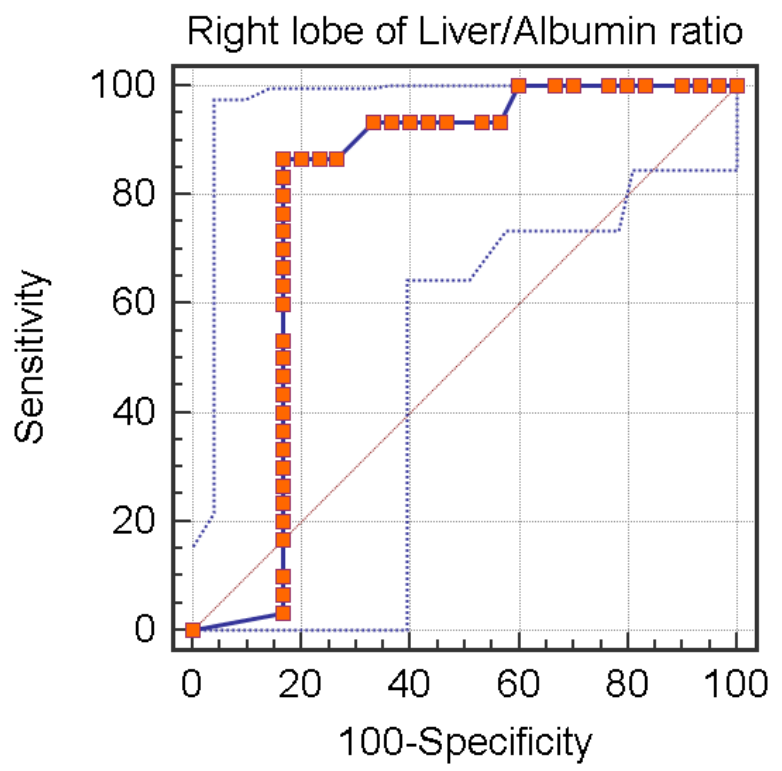

Figure (1) ROC curve between Study and Control regarding right liver lobe/Albumin ratio

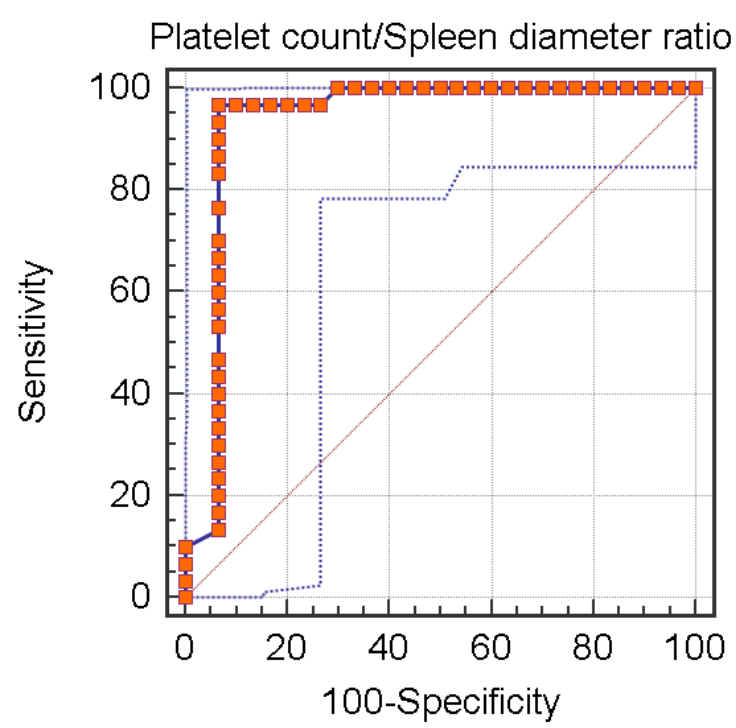

Figure (1): ROC curve between Study and Control regarding Platelet count/spleen diameter ratio.

Table (10): Correlation between Right lobe of Liver/Albumin ratio and Platelet count/Spleen diameter ratio

\begin{tabular}{|c|c|c|}
\hline \multicolumn{3}{|c|}{ Correlations } \\
\hline & \multicolumn{3}{|c|}{ Right lobe of Liver/Albumin ratio } \\
\cline { 2 - 3 } & $\mathbf{R}$ & P-value \\
\hline Platelet count/Spleen diameter ratio & -0.404 & $0.027^{*}$ \\
\hline
\end{tabular}




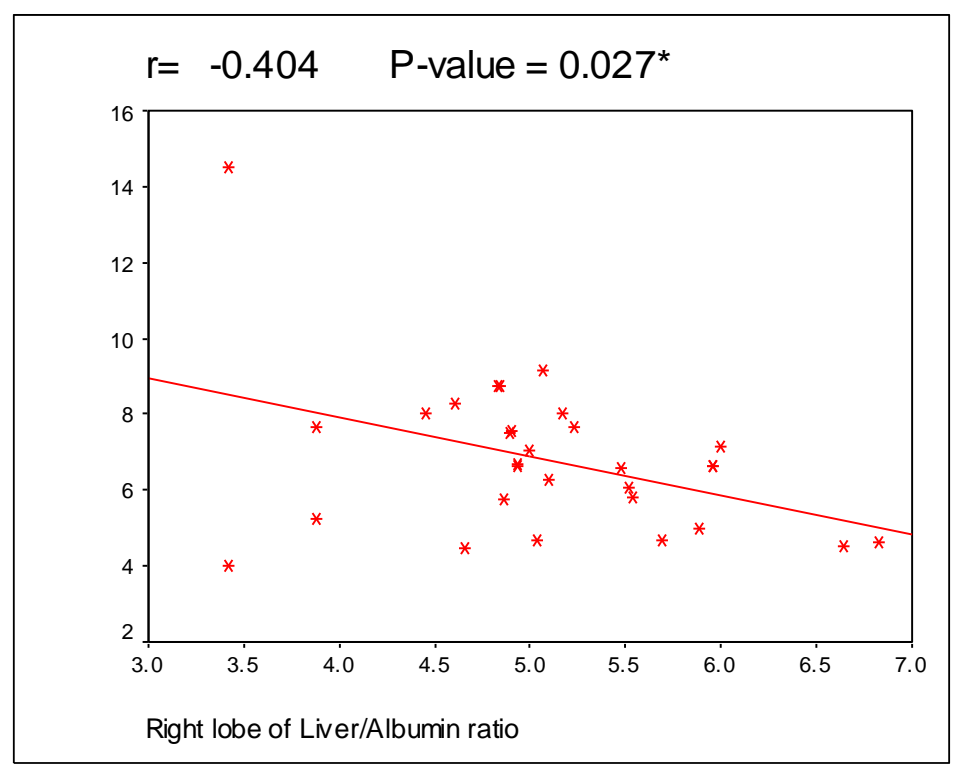

Figure (32): Correlation between Right lobe of Liver/Albumin ratio and Platelet count/Spleen diameter ratio. Table 10 and figure 3 showed that there was a significant negative correlation between the Right lobe of Liver/Albumin ratio and Platelet count/Spleen diameter ratio ( $\mathrm{p}$-value $=0.027$ ).

\section{DISCUSSION}

Oesophageal varices are the most critical porto-systemic shunts that develop secondary to portal hypertension, which is considered the main complication of liver cirrhosis ${ }^{(5)}$.

This study was conducted on 60 cases (divided into 30 cases as control group \& 30 patients with HCVrelated liver cirrhosis as study group) to evaluate the possibility of using Right liver lobe/serum albumin ratio as a non-invasive predictor of esophageal varices in cirrhotic patients due to $\mathrm{HCV}$, for restriction of performance of screening endoscopy.

Regarding the presence of Oesophageal varices (OV) in the study group, we found that 6 patients have grade I OV, 8 patients have grade II, 9 patients have grade III while 7 patients have grade IV OV.

In this study liver enzymes (AST \& ALT), Serum Bilirubin (Total, Direct\& indirect), Prothrombin time, INR, and AFP were higher in the study group than in the control group with highly statistically significant difference $(p=<0.001)$.

But the serum albumin level was lower in the study group than in the control group with a highly statistically significant difference $(p=<0.001)$. Also, we found that serum albumin is decreased as the grade of varices increased.

These results were similar to the results of some researchers ${ }^{(17,18)}$ which can be explained by the fact that the rate of albumin synthesis is reduced up to $50 \%$ in chronic liver disease. Also, serum albumin is reduced in cirrhosis due to an elevated distribution volume in haemodilution, particularly in association with ascites ${ }^{(\mathbf{1 9})}$.
In this study, WBCs, RBCs, and hemoglobin levels showed a non-significant difference between the control and study groups.

But the platelet count was highly statistically significantly decreased in the study group than in the control group ( $p=<0.001)$. Also, the platelet count is decreased as the grade of varices increased with a highly significant value $(p=<0.001)$. This goes in line with what has been reported in a study by Gana $\boldsymbol{e t} \boldsymbol{a l}$. (20) that showed that platelet count is significantly decreased in cirrhotic patients with OV.

Also in our study Spleen diameter (SD) increased in the study group than in the control group with a highly statistically significant difference ( $\mathrm{p}=$ $<0.001$ ), and the spleen diameter increased with the increase of the grade of varices with a high statistically significant difference $(\mathrm{p}=<0.001)$. This comes in agreement with another study by Esmat $\boldsymbol{e t}$ al. ${ }^{(21)}$ that reported a high statistically significant correlation between the presence and grade of oesophageal varices with the splenic diameter.

The right liver lobe diameter/serum albumin (RLLD/Alb) ratio was significantly increased in the study group than in the control group $(\mathrm{P}=<0.005)$. But there was no statistically significant difference between both groups as regard grades of varices.

In this study, the sensitivity \& specificity of the RLLD/Alb ratio was $86.67 \%$ \& $73.33 \%$ respectively and the accuracy of the test was $79.9 \%$, positive predictive value 76.5 , negative predictive value 84.6 with the best cut off point value at $>3.88$.

In comparison to the other similar studies, Alempijevic and his colleagues ${ }^{(19)}$ found that RLLD/albumin ratio can be used for the determination of the presence of OV with a sensitivity of $83.1 \%$ and 
the specificity of $73.9 \%$ with a cut-off value of 4.425 which is nearly similar to our study.

Also, our results go in line with the results by Sanjay and Chandrashekar ${ }^{(22)}$ who found that the sensitivity of the RLLD/Alb ratio was $83.3 \%$ and the specificity was $29.5 \%$ with the best cut off point value 4.42 .

Sheta et al. ${ }^{(23)}$ also supported our results with his colleagues who found that RLLD/albumin at a cutoff value of $\geq 4.92$ significantly predicted the presence of EV with $63.61 \%$ sensitivity, $97.67 \%$ specificity, PPV of $97.3 \%$, and an NPV of $66.7 \%$. The same as in results by Charan et al. (24) who found that the sensitivity of RLLD/Alb ratio was 74.4, specificity 94.4, cut-off value 4.27, PPV was $98.4 \%$, NPV was $44.7 \%$ and accuracy was $78 \%$.

As regard platelet count/spleen diameter (PC/SD) ratio we found that it was decreased in cirrhotic patients with $\mathrm{OV}$ than in the control group with a highly statistically significant difference ( $p=$ $<0.001$ ) (Table 2, Fig. 19). Also, the PC/SD ratio was decreasing as grades of varices was increasing with a highly significant value ( $p=<0.001)$ (table 9, fig. 25). The sensitivity of PC/SD ratio was $96.67 \%$, specificity was $83.33 \%$ and the accuracy of the test was $93.4 \%$, positive predictive value was 85.3 , negative predictive value was 96.2 with best cutoff $\leq 1415$ as in (table 10 , fig. 27).

This comes in agreement with a study by Charan et al. (24) found that the sensitivity of PC/SD ratio was $90.2 \%$, specificity was $88.9 \%$, PPV was $97.4 \%$, NPV was $66.7 \%$ and accuracy was $90 \%$, with the best cut-off value at 1167.2 (AUC 0.965).

Finally, a comparison between RLLD/Alb ratio and $\mathrm{PC} / \mathrm{SD}$ ratio in predicting the presence of $\mathrm{OV}$, showed that the PC/SD ratio had more sensitivity, specificity, and accuracy than did RLLD/Alb ratio, and the correlation between the RLLD/Alb ratio and PC/SD ratio, showed a significant negative correlation between them $(r=-0.404, P=0.027)$.

\section{CONCLUSION}

The use of Right liver lobe size/serum albumin ratio (with or without using platelet count/spleen diameter ratio) can help physicians as a non-invasive predictor of esophageal varices to restrict the use of endoscopic screening only to patients presenting a high probability of esophageal varices. This is especially useful in clinical settings where resources are limited and endoscopic facilities are not present in all areas.

\section{REFERENCES}

1. Shaheen A, Nguyen H, Congly $S$ et al. (2019): Nationwide estimates and risk factors of hospital readmission in patients with cirrhosis in the United States. Liver Int., 39(5):878-884.

2. Yoon H, Shin H, Kim M et al. (2019): Predicting gastroesophageal varices through spleen magnetic resonance elastography in pediatric liver fibrosis. World J Gastroenterol., 25(3):367-377.

3. Nery F, Correia S, Macedo C et al. (2019): Nonselective beta-blockers and the risk of portal vein thrombosis in patients with cirrhosis: results of a prospective longitudinal study. Aliment Pharmacol Ther., 49(5):582-588.

4. Nigatu A, Yap J, Lee Chuy K et al. (2019): Bleeding risk of transesophageal echocardiography in patients with esophageal varices. J Am Soc Echocardiogr., 32(5):674-676.e2.

5. D'Amico G, Pasta L, Morabito A et al. (2014): Competing risks and prognostic stages of cirrhosis: a 25year inception cohort study of 494 patients. Aliment Pharmacol Ther., 39(10):1180-93.

6. Wang L, Hu J, Dong S et al. (2014): Noninvasive prediction of large esophageal varices in liver cirrhosis patients. Clin Invest Med., 37(1): 38-46.

7. Garcia-Tsao G, Abraldes J, Berzigotti A et al. (2017): Portal hypertensive bleeding in cirrhosis: Risk stratification, diagnosis, and management: 2016 practice guidance by the American Association for the study of liver diseases. Hepatology, 65:310-335.

8. Konishi Y, Nakamura T, Kida H et al. (2002): Catheter US probe EUS evaluation of gastric cardia and perigastric vascular structures to predict esophageal variceal recurrence. Gastrointest Endosc., 55:197-203.

9. Lee Y, Chan F, Ching J et al. (2002): Diagnosis of gastroesophageal varices and portal collateral venous abnormalities by endosonography in cirrhotic patients. Endoscopy, 34:391-398.

10. McCarty T, Afinogenova Y, Njei B (2017): Use of Wireless Capsule Endoscopy for the Diagnosis and Grading of Esophageal Varices in Patients With Portal Hypertension: A Systematic Review and MetaAnalysis. J Clin Gastroenterol., 51:174-182.

11. Annicchiarico B, Riccioni $M$, Siciliano $M$ et al. (2019): A pilot study of capsule endoscopy after a standard meal for the detection and grading of oesophageal varices in cirrhotic patients. Dig Liver Dis., 46:997-1000.

12. Laurain A, de Leusse A, Gincul $R$ et al. (2014): Oesophageal capsule endoscopy versus esophagogastroduodenoscopy for the diagnosis of recurrent varices: a prospective multicentre study. Dig Liver Dis., 46:535-540.

13. Ding $\mathbf{N}$, Nguyen $T$, Iser $D$ et al. (2016): Liver stiffness plus platelet count can be used to exclude high-risk oesophageal varices. Liver Int., 36:240-245.

14. de Franchis $R$, Baveno $V$ (2015): Expanding consensus in portal hypertension: Report of the Baveno VI Consensus Workshop: Stratifying risk and individualizing care for portal hypertension. J Hepatol., 63:743-752.

15. Puente A, Hernández-Gea V, Graupera I et al. (2016): Drugs plus ligation to prevent rebleeding in cirrhosis: an updated systematic review. Liver Int., 34(6):823-33.

16. Garcia-Tsao G, Sanyal A, Grace N et al. (2007): Prevention and management of gastroesophageal varices and variceal hemorrhage in cirrhosis. Hepatology, 46(3):922-38. 
17. Said E, Elsayed E, Ameen A et al. (2010): Cytopenia as A Predictor of Oesophageal Varices in Patients with Liver Cirrhosis. Report and Opinion J., 2(7): 35-41.

18. Husová L, Husa $P$, Ovesná $P$ (2011): The influence of some factors on the presence of varices and variceal bleeding in liver cirrhosis patients. Vnitr Lek., 57 (1):6171.

19. Alempijevic L, Bulat V, Djuranovic S (2007): Right liver lobe/albumin ratio: contribution to a non-invasive predictor of Oesophageal varices. World Journal of Gastroenterology, 13(40): 5331-5335.

20. Gana J, Turner D, Roberts E et al. (2010): Derivation of a clinical prediction rule for the noninvasive diagnosis of varices in children. J.Pediatr. Gastroenterol. Nutr., 50(2):188-93.

21. Esmat S, Omarn D and Rashid L(2012): Can we consider the right hepatic lobe size/albumin ratio a non- invasive predictor of oesophageal varices in hepatitis C virus-related liver cirrhotic Egyptian patients? Eur J Intern Med., 23(3):267-72.

22. Sanjay R, Chandrashekar M (2015): Non-invasive prediction of esophageal varices using right lobe of the liver to albumin ratio. International Journal of Recent Trends in Science and Technology, 16(1): 13-15.

23. Sheta $\mathrm{E}$, Yousef $M$, Abd-Elsalam $S$ et al. (2016): Noninvasive diagnosis of esophageal varices: can it replace screening endoscopy? Int J. Curr Microbiol App Sci., 5(5):701-15.

24. Charan S, Garg R, Chander $\mathrm{R}$ et al. (2017): A comparative evaluation of liver lobe size/albumin ratio and platelet count/spleen diameter ratio as non-invasive predictors of oesophageal varices in patients with liver cirrhosis. J Evolution Med Dent Sci., 6(23): 1858-1866. 